\title{
INTERPLAY OF SIMPLE AND SELFADJOINT-IDEAL SEMIGROUPS IN $B(H)$
}

\author{
Sasmita Patnaik And Gary Weiss*
}

Abstract. This paper investigates a question of Radjavi: Which multiplicative semigroups in $B(H)$ have all their ideals selfadjoint (called herein selfadjoint-ideal (SI) semigroups)? We proved this property is a unitary invariant for $B(H)$-semigroups, which invariant we believe is new.

We characterize those SI semigroups $\mathscr{S}$ singly generated by $T$, for $T$ a normal operator and for $T$ a rank one operator. When $T$ is nonselfadjoint and normal or rank one: $\mathscr{S}$ is an SI semigroup if and only if it is simple, except in one special rank one partial isometry case when our characterization yields $\mathscr{S}$ that are SI but not simple. So SI and simplicity are not equivalent notions. When $T$ is selfadjoint, it is straightforward to see that $\mathscr{S}$ is always an SI semigroup, but we prove by examples they may or may not be simple, but for this case we do not have a characterization.

The study of SI semigroups involves solving certain operator equations in the semigroups. A central theme of this paper is to study when and when not SI is equivalent to simple.

Mathematics subject classification (2020): Primary: 47B15, 20M12, 47D03, 20M14; Secondary: 47L20. Keywords and phrases: Semigroups, selfadjoint semigroups, selfadjoint-ideal semigroups, simple semigroups, singly generated semigroups.

\section{REFERENCES}

[1] Janez Bernik, Laurent W. Marcoux, Alexey I. Popov, And Heydar Radjavi, On selfadjoint extensions of semigroups of partial isometries, Trans. Amer. Math. Soc., 368 (11): 7681-7702, 2016.

[2] JANeZ BERniK And AleXey I. Popov, Obstructions for semigroups of partial isometries to be selfadjoint, Math. Proc. Cambridge Philos. Soc., 161 (1): 107-116, 2016.

[3] K. S. CARmen, Semigroup ideals, Master's thesis, University of Tennessee, 8, 1949.

[4] J. L. B. COOPER, One-parameter semigroups of isometric operators in Hilbert space, Ann. of Math. (2), 48: 827-842, 1947.

[5] J. DunCAN And A. L. T. PATERson, $C^{*}$-algebras of inverse semigroups, Proc. Edinburgh Math. Soc. (2), 28 (1): 41-58, 1985.

[6] C. K. Fong And H. Radjavi, On ideals and Lie ideals of compact operators, Math. Ann., 262 (1): 23-28, 1983.

[7] I. GOHBERG AND M. KREIN, Introduction to the Theory of Linear Nonselfdajoint Operators, Translations of Mathematical Monographs.

[8] J. A. GREEn, On the structure of semigroups, Ann. of Math. (2), 54: 163-172, 1951.

[9] P. R. Halmos And L. J. Wallen, Powers of partial isometries, J. Math. Mech., 19: 657-663, 1969/1970.

[10] Paul Richard Halmos, A Hilbert space problem book, Volume 19 of Graduate Texts in Mathematics, Springer-Verlag, New York-Berlin, second edition, 1982. Encyclopedia of Mathematics and its Applications, 17.

[11] Jan OKnińs Ki, Semigroups of matrices, Volume 6 of Series in Algebra. World Scientific Publishing Co., Inc., River Edge, NJ, 1998. 
[12] MATJAŽ OMLAdič AND Heydar RADJAVI, Nilpotent commutators and reducibility of semigroups, Linear Multilinear Algebra, 57 (3): 307-317, 2009.

[13] Matjaž OMLAdič AND Heydar RADJAVI, Reducibility of semigroups and nilpotent commutators with idempotents of rank two, Ars Math. Contemp., 3 (1): 99-108, 2010.

[14] MATJAŽ OMLAdiČ AND Heydar RADJAVI, Self-adjoint semigroups with nilpotent commutators, Linear Algebra Appl., 436 (7): 2597-2603, 2012.

[15] AlAn L. T. PATERSON, Groupoids, inverse semigroups, and their operator algebras, Volume 170 of Progress in Mathematics, Birkhäuser Boston, Inc., Boston, MA, 1999.

[16] Alexey I. Popov and Heydar Radjavi, Semigroups of Partial Isometries, Semigroup Forum (2013) 87: 663-678, https://link.springer.com/article/10.1007/s00233-013-9487-6.

[17] Heydar Radjavi, Polynomial conditions on operator semigroups, J. Operator Theory, 53 (1): $197-$ 220, 2005.

[18] Heydar Radjavi and Peter Rosenthal, Invariant Subspaces, Springer-Verlag, BerlinHeidelberg-New York, 1973.

[19] Heydar Radjavi And Peter Rosenthal, Simultaneous triangularization, Universitext, Springer-Verlag, New York, 2000.

[20] Heydar Radjavi and Peter Rosenthal, Invariant subspaces, Dover Publications, Inc., Mineola, NY, second edition, 2003.

[21] Heydar Radjavi, Peter Rosenthal, and Victor Shulman, Operator semigroups with quasinilpotent commutators, Proc. Amer. Math. Soc., 128 (8): 2413-2420, 2000.

[22] M. H. Stone, On one-parameter unitary groups in Hilbert space, Ann. of Math. (2), 33 (3): 643-648, 1932. 